\title{
Nonlinear heat transport in mesoscopic conductors: Rectification, Peltier effect, and Wiedemann-Franz law
}

\author{
Rosa López and David Sánchez \\ Institut de Física Interdisciplinària i de Sistemes Complexos IFISC (UIB-CSIC), E-07122 Palma de Mallorca, Spain \\ and Departament de Física, Universitat de les Illes Balears, E-07122 Palma de Mallorca, Spain
}

(Received 20 December 2012; published 25 July 2013)

\begin{abstract}
We investigate nonlinear heat properties in mesoscopic conductors using a scattering theory of transport. Our approach is based on a leading-order expansion in both the electrical and thermal driving forces. Beyond linear response, the transport coefficients are functions of the nonequilibrium screening potential that builds up in the system due to interactions. Within a mean-field approximation, we self-consistently calculate the heat rectification properties of a quantum dot attached to two terminals. We discuss nonlinear contributions to the Peltier effect and find departures from the Wiedemann-Franz law in the nonlinear regime of transport.
\end{abstract}

DOI: 10.1103/PhysRevB.88.045129

PACS number(s): 73.23.-b, 73.50.Lw, 73.63.Kv, 73.50.Fq

\section{INTRODUCTION}

Much recent work has raised interest in fundamental questions on energy transport and heat flow at the nanoscopic scale where quantum effects become dominant. ${ }^{1,2}$ Mesoscopic conductors are particularly suitable for investigations of nonequilibrium phenomena in quantum systems since electron transport can be created, manipulated, and detected thanks to the use of electric biases or thermal gradients applied to electronic terminals coupled to the mesoscopic sample. In this way, thermovoltages generated in response to a temperature difference have been observed in quantum point contacts, ${ }^{3}$ quantum dots, ${ }^{4,5}$ and ballistic microjunctions. ${ }^{6}$ These experiments can be successfully explained using linear-response theoretical models. ${ }^{7}$ Much more scarce are contributions that explore the nonlinear regime of transport. 8,9 We have recently proposed in Ref. 10 a general scattering theory of transport for mesoscopic thermoelectric effects in the weakly nonlinear regime taking into account screening interactions out of equilibrium. A natural development of our theory would aim at considering nonlinear effects in the heat current flowing through a mesoscopic system. This is the goal of the present work.

Heat currents can be driven by electric voltages or temperature differences. In the former case, a heat transfer accompanies an electric current, which in the limit of low voltage gives rise to the Peltier effect; in the latter case, a thermal gradient produces a heat flux, which in the limit of small temperature differences is well described by the Fourier law. Nonlinear effects in mesoscopic systems demand the application of large driving forces across small distances, a requirement within the scope of today's techniques. ${ }^{11}$ Nonlinearities have been predicted to cause thermal rectification effects ${ }^{12-14}$ and low temperature cooling. ${ }^{15}$ A deep understanding of nonlinear effects is also needed for a careful assessment of the device performance of heat engines, ${ }^{16}$ heat pumps, ${ }^{17,18}$ and multiterminal heat-to-electric current converters. ${ }^{19,20}$ Our theory is based on a voltage and temperature expansion around the equilibrium state. To lowest order, the linear response coefficients depend on the electrostatic potential at equilibrium only. Higher order terms in the expansion define weakly nonlinear transport coefficients that are functions of the potential landscape out of equilibrium. ${ }^{21}$ This dependence cannot be neglected and is crucial to formulate a gaugeinvariant theory. ${ }^{22}$ We determine the screening potential up to the first order in the driving forces. ${ }^{10}$ In the isothermal case, charge pile-up processes are governed by particle injectivities. Importantly, these injectivities break the Onsager symmetry under reversal of an external magnetic field, ${ }^{23}$ which has been confirmed experimentally. ${ }^{24-28}$ In the isoelectric case, charge can be injected to or from the system depending on whether the carrier's energy lies above or below the chemical potential. Thus, we define entropic injectivities ${ }^{10}$ that specify the charge pile-up in response to a pure thermal gradient. The electrostatic potential is then completely determined once the bare and the screening charges are calculated. As a result, the transmission function becomes a function of energy, voltage, and temperature shifts. ${ }^{10}$ Below, we discuss the consequences of this important result for the nonlinear regime of heat transport.

We examine two applications of our theory. First, we consider nonlinear contributions to the Peltier coefficient. Strikingly enough, there are very few works devoted to the Peltier effect beyond linear response. Exceptions are Ref. 29 on metallic constrictions, Ref. 30 on quantum point contacts, and Ref. 31 on bulk semiconductors. We consider a quantum dot attached to two terminals and calculate self-consistently the Peltier coefficient for higher-order currents. Interestingly, we find that the weakly nonlinear contributions are expressed in terms of a ratio difference that quantifies the relative importance of the nonlinear conductances with respect to the linear ones. Additionally, we test our analytical results with numerical calculations of the full model.

Second, we examine departures of the Wiedemann-Franz law out of equilibrium. We recall that this rule establishes a proportionality relationship between the linear thermal and electric conductances and that the proportionality factor, the Lorenz number, depends on universal physical constants only. Naturally, the Wiedemann-Franz law is not valid outside the Sommerfeld theory of metals (noninteracting electrons and linear-response regime), although it has been empirically found to be satisfied in bulk metals within a wide range of temperatures. Violations of this law in mesoscopic systems 
have been investigated in artificial Kondo impurities, ${ }^{32,33}$ in the fluctuating dynamics of open quantum dots, ${ }^{34}$ in singleelectron transistors, ${ }^{35}$ in strongly interacting dots coupled to ferromagnetic leads, ${ }^{36}$ and in double quantum dots. ${ }^{37}$ Here, we discuss deviations from the Wiedemann-Franz law that emerge in the nonlinear regime of transport only. We find that these departures become maximal when the distance between the Fermi energy and the dot level is of the order of the level broadening because in that case the dot transmission energy dependence becomes strongest.

\section{THEORETICAL MODEL}

Our system consists of a mesoscopic conductor attached to multiple terminals $\alpha, \beta$... Each terminal is electrically and thermally biased as $\mu_{\alpha}=E_{F}+e V_{\alpha}$ and $\theta_{\alpha}=T_{\alpha}-T$, respectively, $\mu_{\alpha}$ being the electrochemical potential, $E_{F}$ the Fermi energy, $T_{\alpha}$ the temperature of lead $\alpha$, and $T$ the background temperature. Charge and heat transport is completely characterized by the scattering matrix $s_{\alpha \beta}=s_{\alpha \beta}(E, e U)$. Generally, the scattering matrix depends on the carrier energy $E$ and the electrostatic potential inside the sample $U .^{21,22}$ The internal potential $U=U\left(\vec{r},\left\{V_{\gamma}\right\},\left\{\theta_{\gamma}\right\}\right)$ is a function of the position $\vec{r}$ and the set of applied voltages $\left\{V_{\gamma}\right\}$ and temperature gradients $\left\{\theta_{\gamma}\right\} .{ }^{10}$ Therefore, a complete calculation of $s_{\alpha \beta}$ as a function of the full potential landscape $U$ in the presence of interactions out of equilibrium seems an insurmountable task. We will then focus on the weakly nonlinear transport regime for which the transport coefficients and the system's response can be expressed in terms of quantities evaluated at equilibrium. ${ }^{21}$

We denote with $\mathcal{I}_{\alpha}$ and $\mathcal{J}_{\alpha}$ the charge and heat currents, respectively, that flow from the leads toward the sample:

$$
\begin{aligned}
I_{\alpha} & =\frac{2 e}{h} \sum_{\beta} \int d E A_{\alpha \beta}(E, e U) f_{\beta}(E), \\
\mathcal{J}_{\alpha} & =\frac{2}{h} \sum_{\beta} \int d E\left(E-\mu_{\alpha}\right) A_{\alpha \beta}(E, e U) f_{\beta}(E),
\end{aligned}
$$

where $\quad A_{\alpha \beta}=\operatorname{Tr}\left[\delta_{\alpha \beta}-s_{\alpha \beta}^{\dagger} s_{\alpha \beta}\right] \quad$ and $\quad f_{\beta}(E)=1 /(1+$ $\left.\exp \left[\left(E-E_{F}-e V_{\beta}\right) / k_{B} T_{\beta}\right]\right)$ is the Fermi distribution function in reservoir $\beta$. A treatment of Eq. (1) in terms of interacting Green functions can be achieved following Ref. 38. Our discussion here is entirely based on the scattering approach.

The sum over all heat flows is $\sum_{i} \mathcal{J}_{\alpha}=\sum_{\alpha} \mathcal{J}_{\alpha}^{E}-$ $\sum_{\alpha} \mathcal{I}_{\alpha} V_{\alpha}$, where $\mathcal{J}_{\alpha}^{E}=(2 / h) \sum_{\beta} \int d E E A_{\alpha \beta}(E, e U) f_{\beta}(E)$ is the energy current. For time-independent driving forces, the energy current is conserved ${ }^{39}$ since unitarity of the scattering matrix $\left(\sum_{\alpha} A_{\alpha \beta}=0\right)$ dictates that $\mathcal{J}_{\alpha}^{E}=0$. Heat fluxes thus satisfy the sum rule $\sum_{\alpha}\left(\mathcal{J}_{\alpha}+\mathcal{I}_{\alpha} \mathcal{V}_{\alpha}\right)=0$

Equations (1) and (2) are exact within the scattering approach, Now, in the weakly nonlinear transport regime charge and heat currents can be expanded around the equilibrium state (defined with $V_{\alpha}=0$ and $\theta_{\alpha}=0$ for all $\alpha$ ) up to second order in powers of the driving fields $V_{\alpha}$ and $\theta_{\alpha}$ :

$$
\begin{aligned}
I_{\alpha}= & \sum_{\beta} G_{\alpha \beta} V_{\beta}+\sum_{\beta} L_{\alpha \beta} \theta_{\beta}+\sum_{\beta \gamma} G_{\alpha \beta \gamma} V_{\beta} V_{\gamma} \\
& +\sum_{\beta \gamma} L_{\alpha \beta \gamma} \theta_{\beta} \theta_{\gamma}+2 \sum_{\beta \gamma} M_{\alpha \beta \gamma} V_{\beta} \theta_{\gamma}, \\
\mathcal{J}_{\alpha}= & \sum_{\beta} R_{\alpha \beta} V_{\beta}+\sum_{\beta} K_{\alpha \beta} \theta_{\beta}+\sum_{\beta \gamma} R_{\alpha \beta \gamma} V_{\beta} V_{\gamma} \\
& +\sum_{\beta \gamma} K_{\alpha \beta \gamma} \theta_{\beta} \theta_{\gamma}+2 \sum_{\beta \gamma} H_{\alpha \beta \gamma} V_{\beta} \theta_{\gamma} .
\end{aligned}
$$

The linear-response electric conductance is given by

$$
G_{\alpha \beta}=\frac{2 e^{2}}{h} \int d E A_{\alpha \beta}(E)\left[-\partial_{E} f(E)\right] .
$$

At very low temperature, the Sommerfeld expansion to leading order in $k_{B} T / E_{F}$ yields the simple expression $G \simeq$ $\left(2 e^{2} / h\right) A_{\alpha \beta}\left(E_{F}\right)$. The linear-response thermoelectric conductance is

$$
L_{\alpha \beta}=\frac{2 e}{h T} \int d E\left(E-E_{F}\right) A_{\alpha \beta}(E)\left[-\partial_{E} f(E)\right],
$$

which reduces to $L_{\alpha \beta}=\left.(2 e / h T)\left(\pi^{2} k_{B}^{2} / 3\right) \partial_{E} A_{\alpha \beta}(E)\right|_{E=E_{F}}$ after the Sommerfeld expansion is applied. The linear-response heat current in Eq. (4) is given by the electrothermal conductance

$$
R_{\alpha \beta}=\frac{2 e}{h} \int d E\left(E-E_{F}\right) A_{\alpha \beta}(E)\left[-\partial_{E} f(E)\right],
$$

and the thermal conductance

$$
K_{\alpha \beta}=\frac{2}{h} \int d E \frac{\left(E-E_{F}\right)^{2}}{T} A_{\alpha \beta}(E)\left[-\partial_{E} f(E)\right],
$$

where analytical expressions can be obtained for these conductances considering again the Sommerfeld expansion: $R_{\alpha \beta}=\left.(2 e / h)\left(\pi^{2} k_{B}^{2} T^{2} / 3\right) \partial_{E} A_{\alpha \beta}(E)\right|_{E=E_{F}}$ and $K_{\alpha \beta}=$ $(2 / h)\left(\pi^{2} k_{B}^{2} T / 3\right) A_{\alpha \beta}\left(E_{F}\right)$. Note that the nondiagonal transport coefficients produce electric current from a temperature difference (the thermoelectric conductance $L_{\alpha \beta}$ ) or heat current from a voltage difference (the electrothermal conductance $R_{\alpha \beta}$ ). Due to reciprocity, both effects are connected: $R_{\alpha \beta}=T L_{\alpha \beta}$.

The linear response coefficients in Eqs. (5)-(8) are evaluated at equilibrium and consequently $G_{\alpha \beta}, L_{\alpha \beta}, R_{\alpha \beta}$, and $K_{\alpha \beta}$ are independent of the screening potential $U$. On the contrary, the leading-order nonlinearities (see below) do depend on the applied electrical and thermal shifts through the electrostatic potential $U$. Indeed, this dependence is responsible for rectification effects in both electrical and heat currents. The nonlinear transport coefficients for transport of charge were obtained in Ref. 10. We here find the nonlinear coefficients for the heat current:

$$
R_{\alpha \beta \gamma}=\frac{e^{2}}{h} \int d E \partial_{E} f(E)\left\{\delta_{\alpha \gamma} A_{\alpha \beta}+\delta_{\alpha \beta} A_{\alpha \beta}-\left(E-E_{F}\right)\left(\frac{\partial A_{\alpha \beta}}{\partial e V_{\gamma}}+\frac{\partial A_{\alpha \gamma}}{\partial e V_{\beta}}\right)-\delta_{\beta \gamma}\left[\left(E-E_{F}\right) \frac{\partial A_{\alpha \beta}}{\partial E}+A_{\alpha \beta}\right]\right\}
$$




$$
\begin{aligned}
& K_{\alpha \beta \gamma}=\frac{1}{h} \int d E \partial_{E} f(E) \frac{\left(E-E_{F}\right)^{2}}{T}\left\{\left(\frac{\partial A_{\alpha \beta}}{\partial \theta_{\gamma}}+\frac{\partial A_{\alpha \gamma}}{\partial \theta_{\beta}}\right)+\delta_{\beta \gamma}\left[\frac{\left(E-E_{F}\right)}{T} \frac{\partial A_{\alpha \beta}}{\partial E}+\frac{A_{\alpha \beta}}{T}\right]\right\}, \\
& H_{\alpha \beta \gamma}=\frac{e}{h} \int d E \partial_{E} f(E)\left(E-E_{F}\right)\left\{\left(\frac{\partial A_{\alpha \gamma}}{\partial \theta_{\beta}}+\frac{E-E_{F}}{T} \frac{\partial A_{\alpha \beta}}{\partial e V_{\gamma}}-\delta_{\alpha \gamma} \frac{A_{\alpha \beta}}{T}\right)+\delta_{\beta \gamma}\left[\frac{\left(E-E_{F}\right)}{T} \frac{\partial A_{\alpha \beta}}{\partial E}+\frac{A_{\alpha \beta}}{T}\right]\right\} .
\end{aligned}
$$

Equations (9a)-(9c) are formally the main results of this work. Notably, the nonlinear responses depend not only on $A_{\alpha \beta}$ but also on its change with variations of the set of electrical and thermal shifts $\left\{V_{\gamma}, \theta_{\gamma}\right\}$ due to the screening response of the system.

A reasonable model for the interactions as response to the electrical and thermal biases considers only small deviations away from equilibrium. Hence, the internal potential is expanded as

$$
U=U_{\mathrm{eq}}+\sum_{\alpha} u_{\alpha} V_{\alpha}+\sum_{\alpha} z_{\alpha} \theta_{\alpha},
$$

where $u_{\alpha}=\left(\partial U / \partial V_{\alpha}\right)_{\text {eq }}$ and $z_{\alpha}=\left(\partial U / \partial \theta_{\alpha}\right)_{\text {eq }}$ are the characteristic potentials. These potential susceptibilities are measures of the internal reaction of the conductor in response to an electrical and thermal shift applied to contact $\alpha$. For definiteness, we next consider the case of a homogeneous potential profile independent of the position, although we emphasize that the extension to inhomogeneous fields in our general model is straightforward.

We first evaluate the total charge $q$ of the conductor, which has two contributions: (i) $q_{\text {bare }}$ corresponding to the bare charge injected from lead $\alpha$ and (ii) the screening charge denoted with $q_{\text {scr }}$. The latter corresponds to the charge that builds up inside the conductor in response to the injected charges. ${ }^{21}$ The bare charge $q_{\text {bare }}$ injected from lead $\alpha$ originates from a voltage and a temperature imbalance in that terminal. Therefore, $q_{\text {bare }}$ is decomposed into the particle injectivity $v_{\alpha}^{p}(E)$ contribution $^{21}$ and the entropic injectivity $v_{\alpha}^{p}(E)$ term: ${ }^{10}$

$$
\begin{aligned}
& v_{\alpha}^{p}(E)=\frac{1}{2 \pi i} \sum_{\beta} \operatorname{Tr}\left[s_{\beta \alpha}^{\dagger} \frac{d s_{\beta \alpha}}{d E}\right], \\
& v_{\alpha}^{e}(E)=\frac{1}{2 \pi i} \sum_{\beta} \operatorname{Tr}\left[\frac{E-E_{F}}{T} s_{\beta \alpha}^{\dagger} \frac{d s_{\beta \alpha}}{d E}\right] .
\end{aligned}
$$

Importantly, the contribution to $q_{\text {bare }}$ due to a temperature shift can be either positive or negative depending on whether the energy of the carriers is above or below $E_{F}$. This notorious feature is related with the fact that a heat addition or removal depends on whether the carrier energy $E$ is larger or smaller than $E_{F} .{ }^{16}$ Then, the factor $\left(E-E_{F}\right) / T$ represents the entropy transfer associated to the additional carrier into the conductor. We also note that in Eq. (12) we write $E_{F}$ instead of the chemical potential $\mu(T)$, which is in general temperature dependent if the reservoir's charge density is assumed to be fixed. ${ }^{40}$ However, in experimentally relevant situations one externally fixes the electrochemical potential using, e.g., voltage sources. Obviously, $\mu \simeq E_{F}$ in the limit of very low temperatures. Finally, the total accumulation or depletion bare charge imbalance becomes

$$
q_{\text {bare }}=e \sum_{\alpha}\left(D_{\alpha}^{p} e V_{\alpha}+D_{\alpha}^{e} \theta_{\alpha}\right),
$$

where $D_{\alpha}^{p}=-\int d E v_{\alpha}^{p}(E) \partial_{E} f$, and $D_{\alpha}^{e}=-\int d E v_{\alpha}^{e}(E) \partial_{E} f$ represent the integrated particle and entropic injectivities around the Fermi energy.

The screening charge is calculated from the response of the internal potential, $\Delta U=U-U_{\text {eq }}$, away from the equilibrium state $U_{\text {eq. }}$. We consider the random phase approximation, in which case the screening charge is proportional to the Lindhard function $\Pi, q_{\mathrm{scr}}=e^{2} \Pi \Delta U$, which in the long wavelength limit becomes $\Pi=\int d E D(E) \partial_{E} f{ }^{41}$ with $D=$ $D\left(E_{F}\right)$ the conductor density of states. The set of equations for the characteristic potentials is closed using the Poisson equation. Thus, we relate the out-of-equilibrium net charge $\delta q=q-q_{\text {eq }}$ to $\Delta U=U-U_{\text {eq }}$ through $\nabla^{2} \Delta U=-4 \pi \Delta q$. By employing Eq. (10) and the fact that $V_{\alpha}$ and $\theta_{\alpha}$ shifts are independent, we find a set of equations for the electrical and thermal characteristic potentials:

$$
\begin{aligned}
& -\nabla^{2} u_{\alpha}+4 \pi e^{2} \Pi u_{\alpha}=4 \pi e^{2} D_{\alpha}^{p}, \\
& -\nabla^{2} z_{\alpha}+4 \pi e^{2} \Pi z_{\alpha}=4 \pi e D_{\alpha}^{e} .
\end{aligned}
$$

It is computationally useful to apply the WKB approximation to the nonlinear coefficients Eqs. (9a)-(9c). Notice that this approach has the same range of validity as the long wavelength limit taken above. Therefore, we can make the replacement $\delta / \delta U \rightarrow-e \partial / \partial E$ in Eqs. (9a)-(9c) and the voltage and temperature derivatives, $\partial_{\theta_{\gamma}} A_{\alpha \beta}$ and $\partial_{V_{\gamma}} A_{\alpha \beta}$, are calculated once the characteristic potentials are known since

$$
\begin{gathered}
\partial_{\theta_{\gamma}} A_{\alpha \beta}=z_{\gamma} \delta A_{\alpha \beta} / \delta U \rightarrow-e z_{\gamma} \partial_{E} A_{\alpha \beta}, \\
\partial_{V_{\gamma}} A_{\alpha \beta}=u_{\gamma} \delta A_{\alpha \beta} / \delta U \rightarrow-e u_{\gamma} \partial_{E} A_{\alpha \beta} .
\end{gathered}
$$

Thus, Eqs. (9a)-(9c) become

$$
\begin{aligned}
& R_{\alpha \beta \gamma}=\frac{e^{2}}{h} \int d E \partial_{E} f(E)\left\{\delta_{\alpha \gamma} A_{\alpha \beta}+\delta_{\alpha \beta} A_{\alpha \beta}+\left(E-E_{F}\right)\left(\frac{\partial A_{\alpha \beta}}{\partial E} u_{\gamma}+\frac{\partial A_{\alpha \gamma}}{\partial E} u_{\beta}\right)-\delta_{\beta \gamma}\left[\left(E-E_{F}\right) \frac{\partial A_{\alpha \beta}}{\partial E}+A_{\alpha \beta}\right]\right\}, \\
& K_{\alpha \beta \gamma}=-\frac{1}{h} \int d E \partial_{E} f(E) \frac{\left(E-E_{F}\right)^{2}}{T}\left\{\left(e \frac{\partial A_{\alpha \beta}}{\partial E} z_{\gamma}+e \frac{\partial A_{\alpha \gamma}}{\partial E} z_{\beta}\right)-\delta_{\beta \gamma}\left[\frac{\left(E-E_{F}\right)}{T} \frac{\partial A_{\alpha \beta}}{\partial E}+\frac{A_{\alpha \beta}}{T}\right]\right\},
\end{aligned}
$$




$$
H_{\alpha \beta \gamma}=\frac{-e}{h} \int d E \partial_{E} f(E)\left(E-E_{F}\right)\left\{\left(e \frac{\partial A_{\alpha \gamma}}{\partial E} z_{\beta}+\frac{E-E_{F}}{T} \frac{\partial A_{\alpha \beta}}{\partial E} u_{\gamma}+\delta_{\alpha \gamma} \frac{A_{\alpha \beta}}{T}\right)-\delta_{\beta \gamma}\left[\frac{\left(E-E_{F}\right)}{T} \frac{\partial A_{\alpha \beta}}{\partial E}+\frac{A_{\alpha \beta}}{T}\right]\right\} .
$$

In the low temperature limit and for a two terminal device $(\alpha=1,2)$, the number of nonlinear conductances in Eq. (17) can be greatly reduced because the electrothermal and thermal conductances for contact $\alpha=1$ can be expressed in terms of the characteristic potentials $u_{1,2}$ and $z_{1,2}$ and the total transmission $\mathcal{T}(E)$ as

$$
\begin{aligned}
& \left.R_{111} \approx \frac{e^{2}}{h}\left[-\mathcal{T}(E)+\frac{\pi^{2} k_{B}^{2} T^{2}}{3}\left(1-2 u_{1}\right) \frac{\partial^{2} \mathcal{T}(E)}{\partial E^{2}}\right]\right|_{E=E_{F}}, \\
& \left.K_{111} \approx \frac{e}{h} \frac{\pi^{2} k_{B}^{2} T}{3}\left[2 z_{1} \frac{\partial \mathcal{T}(E)}{\partial E}-\frac{\mathcal{T}(E)}{e T}\right]\right|_{E=E_{F}}, \\
& \left.H_{111} \approx \frac{e^{2}}{h} \frac{\pi^{2} k_{B}^{2} T^{2}}{3}\left[z_{1} \frac{\partial^{2} \mathcal{T}(E)}{\partial E^{2}}+u_{1} \frac{1}{e T} \frac{\partial \mathcal{T}(E)}{\partial E}\right]\right|_{E=E_{F}} .
\end{aligned}
$$

\section{QUANTUM DOT}

In order to illustrate the general formalism described in the previous section, we now investigate the nonlinear heat transport for a paradigmatic mesoscopic system: an interacting quantum dot. Recent experimental findings support interesting nonlinear thermoelectric effects in quantum dots. ${ }^{9}$ We theoretically model the quantum dot system with a single localized level with energy $E_{d}$. The dot is attached to two reservoirs via tunneling barriers as shown in Fig. 1. We model such rates with energy independent constants $\Gamma_{1}$ and $\Gamma_{2}$, resulting in a total dot level broadening given by $\Gamma=\Gamma_{1}+\Gamma_{2}$. We treat the electrostatic interaction within a mean-field description. Under these considerations, the heat current from contact 1 is calculated from

$\mathcal{J}_{1}=\frac{2}{h} \int d E\left(E-E_{F}-e V_{1}\right) \mathcal{T}(E)\left[f_{1}(E)-f_{2}(E)\right]$,

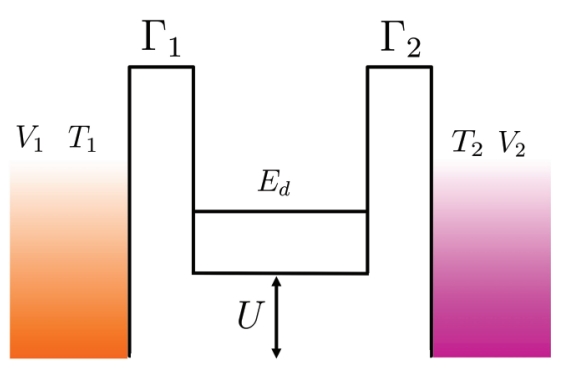

FIG. 1. (Color online) Sketch of a dot system driven out of equilibrium by voltage biases $V_{1}$ and $V_{2}$ and thermal differences $T_{1}$ and $T_{2} . \Gamma_{1}$ and $\Gamma_{2}$ denote the reservoir-dot tunneling rates to the external reservoirs and $E_{d}$ represents the dot energy level. $U$ is the internal dot potential illustrated as a shift of the dot bottom band. where

$$
\mathcal{T}=\frac{4 \Gamma_{1} \Gamma_{2}}{\left(E-E_{d}-e U\right)^{2}+\Gamma^{2}}
$$

is the corresponding Breit-Wigner transmission line shape for the dot level as a function of the internal potential $U$. The dot charge reads

$$
q_{d}=\frac{e}{\pi} \int d E \frac{\Gamma_{1} f_{1}(E)+\Gamma_{2} f_{2}(E)}{\left(E-E_{d}-e U\right)^{2}+\Gamma^{2}} .
$$

Within a mean-field treatment of interactions, we solve the discrete version of the Poisson equation by introducing a geometrical capacitance $C$ which connects electrically the dot to an external gate terminal $V_{g}$ controlling the level position. Then, $U$ is determined from

$$
\delta q_{d}=q_{d}-q_{d}^{\mathrm{eq}}=C\left(U-V_{g}\right),
$$

where $\delta q_{d}$ represents the charge excess due to electrical and thermal applied biases while $q_{d}^{\text {eq }}$ is the equilibrium charge calculated from Eq. (21) by setting $f_{1}=f_{2}=f$.

Once the internal dot potential $U$ is obtained, Eq. (19) can be integrated and yields

$$
\mathcal{J}_{1}=\frac{4 \Gamma_{1} \Gamma_{2}}{\Gamma \hbar} \operatorname{Im}\left\{\beta_{1} \xi_{1}\left[\Psi\left(\frac{1}{2}+i \frac{\xi_{2}}{\pi}\right)-\Psi\left(\frac{1}{2}+\frac{i \xi_{1}}{\pi}\right)\right]\right\}
$$

where $\Psi$ is the digamma function with arguments $\xi_{1(2)}=$ $\beta_{1(2)}\left[\tilde{E}_{d}-i \Gamma-\mu_{1(2)}\right]$ where $\beta_{1(2)}=1 / k_{B} T_{1(2)}$. Importantly, the dot level is renormalized by the interactions: $\tilde{E}_{d}=$ $E_{d}+e U$.

Equation (23) is the exact expression for the heat current when Coulomb interaction is considered within a mean-field description. It is to be compared to the voltage-temperature expansion obtained from the nonlinear heat transport formalism discussed in Sec. II. To do so, we calculate the heat transport conductances in terms of the characteristic potentials and expand Eq. (21) to leading order in $V_{\alpha}, \theta_{\alpha}$ and $U$. We find

$\delta q_{d}=e^{2} D_{1}^{p} V_{1}+e^{2} D_{2}^{p} V_{2}+e D_{1}^{e} \theta_{1}+e D_{2}^{e} \theta_{2}-e^{2} D U$,

where

$$
\begin{aligned}
D_{\alpha}^{p} & =\frac{-\Gamma_{\alpha}}{\pi} \int d E \frac{\partial_{E} f(E)}{\left(E-E_{d}\right)^{2}+\Gamma^{2}}, \\
D_{\alpha}^{e} & =\frac{-\Gamma_{\alpha}}{\pi} \int d E \frac{E-E_{F}}{T} \frac{\partial_{E} f(E)}{\left(E-E_{d}\right)^{2}+\Gamma^{2}} .
\end{aligned}
$$

Notice that Eqs. (25) and (26) are the integrated particle and entropic injectivities within a Breit-Wigner representation of the dot scattering matrix. Using Eq. (22) and (24) we find the dot internal potential

$$
U=\frac{e^{2} D_{1}^{p} V_{1}+e^{2} D_{2}^{p} V_{2}+e D_{1}^{e} \theta_{1}+e D_{2}^{e} \theta_{2}+C V_{g}}{C+e^{2} D},
$$


from which the characteristic potentials follow,

$$
u_{1(2)}=\frac{e^{2} D_{1(2)}^{p}}{C+e^{2} D}, \quad u_{g}=\frac{C}{C+e^{2} D}, \quad z_{1(2)}=\frac{e D_{1(2)}^{e}}{C+e^{2} D} .
$$

Note that while $u_{1}+u_{2}+u_{g}=1$ because of gauge invariance $^{21}$ such a sum rule is not satisfied by the $z$ potentials.

\section{HEAT RECTIFICATION EFFECTS}

Rectification effects occur in systems where the functional dependence of current versus the driving field (voltage or temperature shift) departs from being linear due to quadratic (nonlinear) transport responses. Rectification can arise from interaction with electromagnetic environments ${ }^{12}$ or in multiple-level quantum dot systems. ${ }^{13,14}$ Here, we investigate nonlinearities in the heat flux-voltage and heattemperatures characteristics of a single-level quantum dot in the absence of electromagnetic fluctuations. Additionally, we consider the charge neutral limit $(C=0)$, in which case strong interactions renormalize the energy level to maintain a fixed charge inside the dot. This limit is relevant in many experimental situations.

In the isothermal case $\left(T_{1}=T_{2}\right)$, we take $V_{1}=V$ and $V_{2}=$ 0 . Then, to leading order in voltage the characteristic potential becomes $u=\partial U / \partial V=(1+\eta) / 2$ with $\eta=\left(\Gamma_{1}-\Gamma_{2}\right) / \Gamma$ the tunneling asymmetry. ${ }^{23}$ In the upper inset of Fig. 2(a) we show the exact dot potential $U$ obtained from a numerical, self-consistent calculation of Eq. (21) compared to its leadingorder value $U=u V$. We observe that the approximation is rather good even for voltages larger than the level broadening $\Gamma$. The heat current $\mathcal{J} \equiv \mathcal{J}_{1}$ is shown in the main part of Fig. 2 as a function of voltage. At low voltages, the relation is linear but is not clearly visible since the quadratic-in- $V$ term largely dominates (dashed lines). We recall that at low temperatures $\mathcal{J} \simeq R_{111} V^{2}$ represents a Joule heating term, which is the main source of heat release in a voltage-biased terminal. In the lower inset of Fig. 2(a) we plot the nonlinear electrothermal conductance as a function of the background temperature. Interestingly, $R_{111}$ increases in magnitude more quickly as the tunneling asymmetry increases.

In the isoelectric case $\left(\mu_{1}=\mu_{2}\right)$, we take $\theta_{1}=\theta$ and $\theta_{2}=$ 0 . The characteristic potential determined from the entropic injectivities is $z=\partial U / \partial \theta=D_{1}^{e} /\left[2 e\left(D_{1}^{p}+D_{2}^{p}\right)\right]$. Thus, the dot potential reduces to $U=z \theta$ to leading order in $\theta$. We compare in the upper inset of Fig. 2(b) the validity of this approximation with the exact calculation of $U$. We find that for thermal shifts much smaller than $\Gamma$, the relation between $U$ and $\theta$ stays linear. In the main part of Fig. 2(b) we depict the heat current as function of $\theta$ for various values of the tunneling asymmetry. Since we select small values of $\theta$, the agreement between the weakly nonlinear expressions and the full calculation is excellent. In the lower inset of Fig. 2(b) we show the nonlinear thermal conductance $K_{111}$ as a function of the background temperature $T$. Similarly to the isothermal case, the rectifying thermal conductance increases with higher values of $T$.
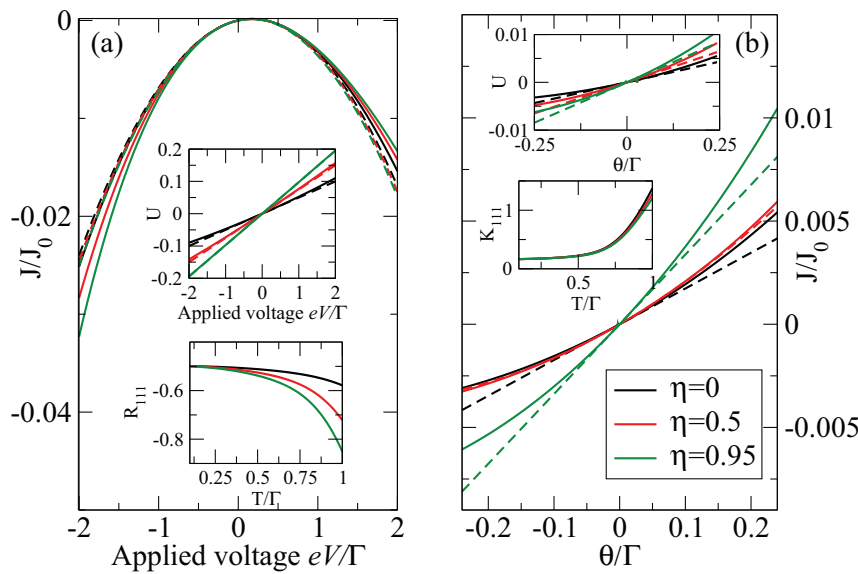

FIG. 2. (Color online) Heat current $\mathcal{J}$ (in units of $\mathcal{J}_{0}=2 \Gamma_{1} \Gamma_{2} / h$ ) for a quantum dot system with $\mu_{1}=E_{F}+e V, \mu_{2}=E_{F}, \theta_{1}=$ $T+\theta$, and $\theta_{2}=T$ for three different values of the tunneling asymmetry $\eta$ and $E_{d}=10 \Gamma$ with $T=0.5 \Gamma$. (a) $\mathcal{J}-V$ characteristics (full lines) for $\theta=0$ along with the leading-order nonlinearity $\mathcal{J} \simeq R_{11} V+R_{111} V^{2}$ (dashed lines) for the $\eta$ values indicated in the right panel. Upper inset: We show with solid lines the self-consistent potential $U$ as a function of $\mathrm{eV} / \Gamma$. Dotted lines correspond to the approximated values $U \approx u V=\Gamma_{1} / \Gamma$ to leading order in an expansion in powers of $V$. Lower inset: temperature dependence of $R_{111}$. (b) $\mathcal{J}-\theta$ characteristics (full lines) for $V=0$ along with the leading-order nonlinearity $\mathcal{J} \simeq K_{11} \theta+K_{111} \theta^{2}$ (dashed lines) for the indicated values of $\eta$. Upper inset: We show with solid lines the self-consistent potential $U$ as a function of the thermal shift. Lower inset: temperature dependence of the nonlinear thermal conductance $K_{111}$.

\section{NONLINEAR PELTIER EFFECT}

The Peltier effect accounts for the generated heat in a given terminal caused by the flow of a unit charge through the conductor, keeping all terminals at the same temperature. More precisely, the two-terminal Peltier coefficient is defined at linear response as the ratio between heat and electrical currents,

$$
\Pi_{0}=\frac{\mathcal{J}_{1}}{\mathcal{I}_{1}}=\frac{R_{11}}{G_{11}},
$$

when $\theta_{\gamma}=0$ for all $\gamma$. Thus, the Peltier coefficient can be viewed as the analog to the thermopower $S_{0}$ (a voltage generation in response to a thermal gradient under the condition of zero net current). In fact, both coefficients are connected via the Kelvin relation $\Pi_{0}=T S_{0}$. The Peltier effect informs us about how efficiently electrical currents are converted into heat currents whereas the Seebeck effect quantifies the transformation of waste heat into useful electricity.

Our goal here is to generalize Eq. (29) to the nonlinear case. We consider the following expression,

$$
\Pi_{\alpha}=\left.\frac{\mathcal{J}_{\alpha}}{\mathcal{I}_{\alpha}}\right|_{\left\{\theta_{\gamma}=0\right\}} .
$$

To calculate the Peltier coefficient in the weakly nonlinear regime we employ the second order expansion in terms of electrical and temperature gradients for both the electrical and heat currents. For a two-terminal device an isothermal 
electrical current $\mathcal{I}_{1}=\mathcal{I}$ is driven by a bias voltage drop $V$,

$$
\mathcal{I}=G_{11} V+G_{111} V^{2}+\mathcal{O}\left(V^{3}\right) .
$$

Inverting this expression, we substitute the voltage $V=$ $\mathcal{I} / G_{11}-\mathcal{I}^{2} G_{111} / G_{11}+\mathcal{O}\left(\mathcal{I}^{3}\right)+\cdots$ into the heat current,

$$
\mathcal{J}_{1}=R_{11} V+R_{111} V^{2}+\mathcal{O}\left(V^{3}\right),
$$

yielding

$$
\mathcal{J}_{1}=\frac{R_{11}}{G_{11}} \mathcal{I}+\frac{1}{G_{11}}\left(\frac{R_{111}}{R_{11}}-\frac{G_{111}}{G_{11}}\right) \mathcal{I}^{2}+\mathcal{O}\left(\mathcal{I}^{3}\right) .
$$

We insert Eq. (33) in Eq. (30) and find

$$
\Pi=\Pi_{0}\left[1+\frac{1}{G_{11}}\left(\frac{R_{111}}{R_{11}}-\frac{G_{111}}{G_{11}}\right) \mathcal{I}+\cdots\right],
$$

where we have set $\Pi \equiv \Pi_{1}$. Now, the second term in the r.h.s of Eq. (34) measures the deviations of $\Pi$ from the linear-response value, $\Pi_{0}$. Interestingly enough, nonlinear contributions are dictated by a ratio between the nonlinear to linear thermal and electrical conductances. In other words, the conversion efficiency for electric currents into heat flow is given by the relative strength of the nonlinear conductances to the linear ones and by the difference between the heat and the electrical properties of the conductor.

We show in Fig. 3(a) the linear Peltier coefficient for a two-terminal quantum dot as described in Sec. II taking $G_{111}=$ $\left.\left(e^{3} / h\right)\left(1-2 u_{1}\right) \partial_{E} \mathcal{T}(E)\right|_{E=E_{F}} .{ }^{21,22}$ As the background $T$ increases, $\Pi_{0}$ quickly enhances following a $T^{2}$ law and then saturates for temperatures much larger than the resonance broadening $\Gamma$. The first fact can be understood from a Sommerfeld expansion of $R_{11}$ and $G_{11}$. The former goes as $T^{2}$ whereas the latter is independent of temperature to leading order in $T$. Finally, the saturation value can be deduced by replacing the Lorentzian shape of the Breit-Wigner resonance with a delta-like peak, an approximation valid in the high temperature regime $(T \gg \Gamma)$. Then, one finds $\Pi_{0}=-\left(E_{0}-E-F\right) / e$. Note that this expression obeys the Kelvin relation $\Pi_{0}=T S_{0}$

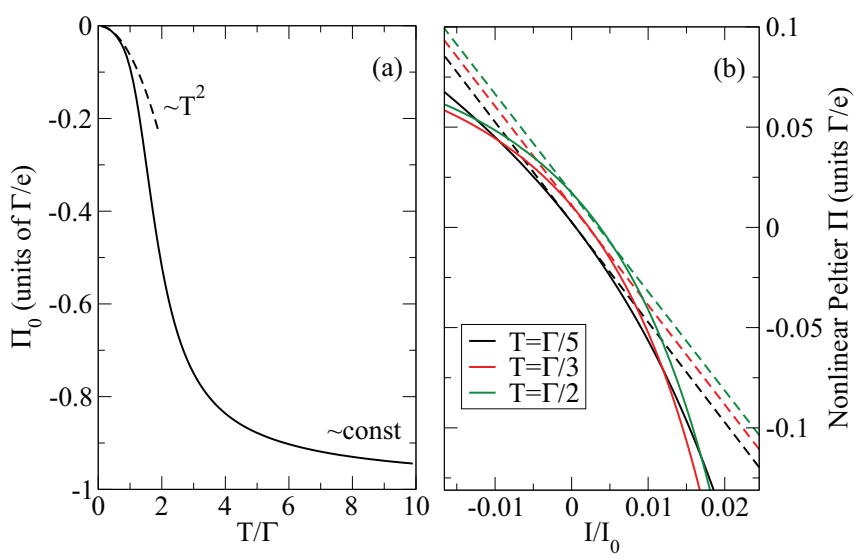

FIG. 3. (Color online) (a) Linear-response Peltier coefficient $\Pi_{0}$ for a current biased quantum dot at $E_{d}=10 \Gamma$. We show both the low and high temperature limits. (b) Full Peltier coefficient $\Pi$ including contributions beyond linear response for three different background temperatures for $\eta=0.5$. We show with colored dotted lines the leading-order expansion calculated from Eq. (34). since the linear-response thermopower for the same system is $S_{0}=-\left(E_{0}-E-F\right) / e T$ at high temperatures. ${ }^{10}$

In the nonlinear regime, $\Pi$ depends on the nonlinear responses according to Eq. (34) and a self-consistent calculation is thus needed. We depict in Fig. 3(b) the exact value of $\Pi$ obtained from a numerical calculation of Eqs. (1), and (2) in the mean-field approach for interactions (full lines), together with the weakly nonlinear result expressed by Eq. (34). To leading order in $\mathcal{I} / \mathcal{I}_{0}$, where $\mathcal{I}_{0}=\left(2 e^{2} / h\right) \Gamma_{1} \Gamma_{2} / \Gamma$, the Peltier coefficient shows a linear dependence of the current, in agreement with Eq. (34). Furthermore, $\Pi$ increases for increasing $\mathcal{I}$ but only in the low current regime. At higher currents, highly nonlinear terms start to contribute and deviations from the linear regime are observed.

\section{WIEDEMANN-FRANZ LAW}

The Wiedemann-Franz law for metals establishes that the ratio between the linear-response thermal $(K)$ and electrical $(G)$ conductances is proportional to the temperature $(T)$ with a proportionality constant given by the Lorenz number, $\Lambda_{0}=$ $\left(\pi^{2} / 3\right)\left(k_{B} / e\right)^{2}$. This relation holds as long as the Sommerfeld expansion is valid. More concisely, the Wiedemann-Franz law for a mesoscopic system states that

$$
\frac{K}{T G}=\Lambda_{0},
$$

which is obviously satisfied in the linear regime since $K \simeq$ $(2 / 3 h) \pi^{2} k_{B}^{2} T \mathcal{T}\left(E_{F}\right)$ and $G \simeq\left(2 e^{2} / h\right) \mathcal{T}\left(E_{F}\right)$.

Deviations from Eq. (35) have been reported before ${ }^{32-37}$ but only within linear response theory. To test the validity of the Wiedemann-Franz law in the nonlinear regime of transport, we here consider the Wiedemann-Franz ratio

$$
\Lambda_{\alpha}=\frac{\left.\left(\mathcal{J}_{\alpha} / \theta\right)\right|_{\{V=0\}}}{\left.T\left(\mathcal{I}_{\alpha} / V\right)\right|_{\{\theta=0\}}}
$$

For illustrative purposes we have considered again the case of a conductor attached to two terminals, where only one of the contacts is heated or cooled with thermal difference $\theta$ and electrically biased with voltage $V$; e.g., $\theta_{1}=\theta, \theta_{2}=0$, $V_{1}=V$, and $V_{2}=0$. Then,

$$
\Lambda=\frac{1}{T} \frac{K_{11}+K_{111} \theta+\cdots}{G_{11}+G_{111} V+\cdots} .
$$

Keeping only terms linear in $\theta$ and $V$, the nonlinear analog to the Wiedemann-Franz ratio is

$$
\Lambda=\frac{1}{T}\left(\frac{K_{11}}{G_{11}}-\frac{K_{11} G_{111} V}{G_{11}^{2}}+\frac{K_{111} \theta}{G_{11}}+\cdots\right) .
$$

Clearly, Eq. (35) reduces to Eq. (38) if nonlinear terms are neglected. Departures from the Wiedemann-Franz rule occurring in the nonlinear regime of transport can thus be quantified with the following magnitude:

$$
\frac{\Lambda-\Lambda_{0}}{\Lambda_{0}}=\frac{K_{111}}{K_{11}} V-\frac{G_{111}}{G_{11}} \theta .
$$

Interestingly, deviations from the Lorenz number are given by the difference between the ratios of nonlinear to linear thermal and electrical conductances. 


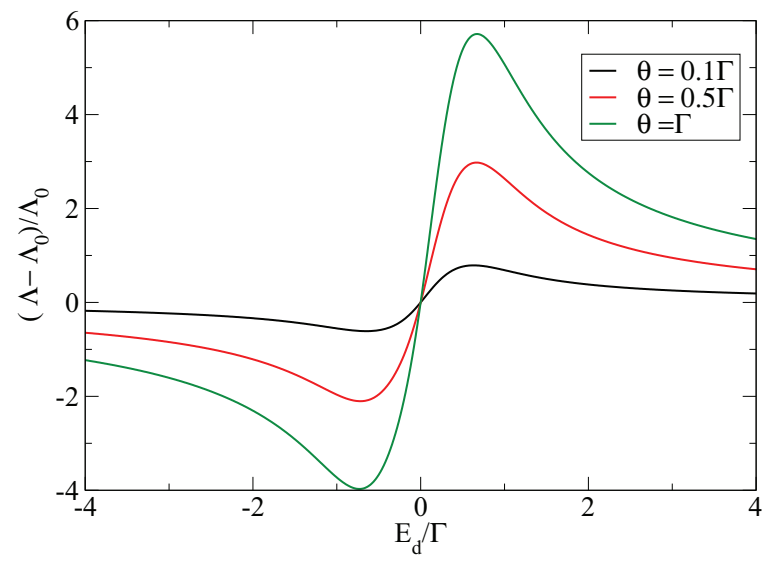

FIG. 4. (Color online) Nonlinear departures of the WiedemannFranz law as expressed by Eq. (39). Nonlinear transport responses are calculated for a two-terminal quantum dot with voltage bias $V=$ $0.25 \Gamma$, background temperature $T=0.1 \Gamma$, and different values of thermal shift $\theta$ as a function of the dot level position $E_{d}$.

We calculate Eq. (39) for the quantum dot model described in Sec. III for $V=0.25 \Gamma$ and $T=0.01 \Gamma$ and different values of $\theta$. We show our results in Fig. 4 as a function of the dot level position $E_{d}$. We find that departures are stronger for increasing temperature differences since for increasing $\theta$ the heat transport becomes more nonlinear. Moreover, when $E_{d}$ lies around $\Gamma$ above or below the Fermi energy departures are more apparent because the transmission function changes rapidly for energies around those points.

\section{CONCLUSIONS}

We have investigated the heat flux in a mesoscopic system beyond linear response. Our scattering approach includes interactions to take into account the system's screening response to voltage and temperature shifts in the reservoirs. We have found exact expressions for the leading-order nonlinearities in a voltage-temperature expansion of the heat current and have applied our general theory to the study of the heat rectification properties of an interacting quantum dot attached to two terminals. We have found that the Peltier coefficient acquires higher-order contributions at moderate electric currents and that these contributions are stronger for increasing background temperature. Furthermore, we have investigated departures from the Wiedemann-Franz law in the weakly nonlinear regime of transport and have found that the strongest deviations occur when charge fluctuations dominate, i.e., when the distance between the dot level and the leads' Fermi energy is of the order of the resonance broadening. We believe that our results are of fundamental importance for the understanding of basic nonlinear heating effects in quantum conductors.

Recently, we became aware of a closely related work by J. Meair and Ph. Jacquod (Ref. 42). The difference is that they are concerned with thermodynamic efficiencies while we focus on the nonlinear Peltier effect and departures from the Wiedemann-Franz law.

\section{ACKNOWLEDGMENTS}

This work was supported by MINECO Grant No. FIS201123526.
${ }^{1}$ A. Dhar, Adv. Phys. 57, 457 (2008)

${ }^{2}$ Y. Dubi and M. Di Ventra, Rev. Mod. Phys. 83, 131 (2011).

${ }^{3}$ L. W. Molenkamp, Th. Gravier, H. van Houten, O. J. A. Buijk, M. A. A. Mabesoone, and C. T. Foxon, Phys. Rev. Lett. 68, 3765 (1992).

${ }^{4}$ A. S. Dzurak, C. G. Smith, C. H. W. Barnes, M. Pepper, L. MartínMoreno, C. T. Liang, D. A. Ritchie, and G. A. C. Jones, Phys. Rev. B 55, R10197 (1997).

${ }^{5}$ S. F. Godijn, S. Möller, H. Buhmann, L. W. Molenkamp, and S. A. van Langen, Phys. Rev. Lett. 82, 2927 (1999).

${ }^{6}$ J. Matthews, D. Sánchez, M. Larsson, and H. Linke, Phys. Rev. B 85, 205309 (2012).

${ }^{7}$ P. N. Butcher, J. Phys.: Condens. Matter 2, 4869 (1990).

${ }^{8}$ A. A. M. Staring, L. W. Molenkamp, B. W. Alphenaar, H. van Houten, O. J. A. Buyk, M. A. A. Mabesoone, C. W. J. Beenakker, and C. T. Foxon, Europhys. Lett. 22, 57 (1993).

${ }^{9}$ S. Fahlvik Svensson, E. A. Hoffmann, N. Nakpathomkun, P. M. Wu, H. Q. Xu, H. A. Nilsson, D. Sánchez, V. Kashcheyevs, and H. Linke, arXiv:1307.0617.

${ }^{10}$ D. Sánchez and R. López, Phys. Rev. Lett 110, 026804 (2013).

${ }^{11}$ R. Venkatasubramanian, E. Siivola, T. Colpitts, and B. O'Quinn, Nature (London) 413, 597 (2001).

${ }^{12}$ T. Ruokola, T. Ojanen, and A.-P. Jauho, Phys. Rev. B 79, 144306 (2009).

${ }^{13}$ David M.-T. Kuo and Y.C. Chang, Phys. Rev. B 81, 205321 (2010).
${ }^{14}$ X. O. Chen, B. Dong, and X. L. Lei, Chin. Phys. Lett. 25, 3032 (2008).

${ }^{15}$ R. S. Whitney, Phys. Rev. B 87, 115404 (2013); arXiv:1208.6130 [Phys. Rev. B (to be published)].

${ }^{16}$ T. E. Humphrey, R. Newbury, R. P. Taylor, and H. Linke, Phys. Rev. Lett. 89, 116801 (2002).

${ }^{17}$ D. Segal and A. Nitzan, Phys. Rev. E 73, 026109 (2006).

${ }^{18}$ M. Moskalets and M. Büttiker, Phys. Rev. B 66, 035306 (2002).

${ }^{19}$ R. Sánchez and M. Büttiker, Phys. Rev. B 83, 085428 (2011).

${ }^{20}$ J.-H. Jiang, O. Entin-Wohlman, and Y. Imry, Phys. Rev. B 85, 075412 (2012)

${ }^{21}$ M. Büttiker, J. Phys. Condens. Matt. 5, 9361 (1993).

${ }^{22}$ T. Christen and M. Büttiker, Europhys. Lett. 35, 523 (1996).

${ }^{23}$ D. Sánchez and M. Büttiker, Phys. Rev. Lett. 93, 106802 (2004); Phys. Rev. B 72, 201308(R) (2005).

${ }^{24}$ C. A. Marlow, R. P. Taylor, M. Fairbanks, I. Shorubalko, and H. Linke, Phys. Rev. Lett. 96, 116801 (2006)

${ }^{25}$ R. Leturcq, D. Sánchez, G. Götz, T. Ihn, K. Ensslin, D. C. Driscoll, and A. C. Gossard, Phys. Rev. Lett. 96, 126801 (2006).

${ }^{26}$ D. M. Zumbühl, C. M. Marcus, M. P. Hanson, and A. C. Gossard, Phys. Rev. Lett. 96, 206802 (2006).

${ }^{27}$ L. Angers, E. Zakka-Bajjani, R. Deblock, S. Gueron, H. Bouchiat, A. Cavanna, U. Gennser, and M. Polianski, Phys. Rev. B 75, 115309 (2007). 
${ }^{28}$ D. Hartmann, L. Worschech, and A. Forchel, Phys. Rev. B 78, 113306 (2008).

${ }^{29}$ I. O. Kulik, J. Phys.: Condens. Matter 6, 9737 (1994).

${ }^{30}$ E. N. Bogachek, A. G. Scherbakov, and U. Landman, Phys. Rev. B 60, 11678 (1999).

${ }^{31}$ M. Zebarjadi, K. Esfarjani, and A. Shakouri, Appl. Phys. Lett. 91, 122104 (2007).

${ }^{32}$ D. Boese and R. Fazio, Europhys. Lett. 56, 576 (2001).

${ }^{33}$ B. Dong and X. L. Lei, J. Phys.: Condens. Matter 14, 11747 (2002).

${ }^{34}$ M. G. Vavilov and A. D. Stone, Phys. Rev. B 72, 205107 (2005).
${ }^{35}$ B. Kubala, J. König, and J. Pekola, Phys. Rev. Lett. 100, 066801 (2008).

${ }^{36}$ M. Krawiec and K. I. Wysokiński, Phys. Rev. B 73, 075307 (2006).

${ }^{37}$ P. Trocha and J. Barnaś, Phys. Rev. B 85, 085408 (2012).

${ }^{38}$ Tai Kai Ng and Patrick A. Lee, Phys. Rev. Lett. 61, 1768 (1988).

${ }^{39}$ G. D. Mahan, Many-Particle Physics, 3rd. ed. (Kluwer, New York, 2000), p. 25.

${ }^{40}$ N. W. Ashcroft and N. D. Mermin, Solid State Physics (Saunders College, Philadelphia, PA, 1976), Chap. 2.

${ }^{41}$ H. Smith, Phys. Scr. 28, 287 (1983).

${ }^{42}$ J. Meair and Ph. Jacquod, J. Phys. Cond. Mat. 25, 082201 (2013). 